\title{
AVALIAÇÃO DOS RISCOS PSICOSSOCIAIS NO CENTRO DE MATERIAL E ESTERILIZAÇÃO DO NORTE DO BRASIL
}

\author{
Evaluation of psychosocial risks in the central sterile supply department of northern Brazil
}

\section{Evaluación de riesgos psicosociales en el centro de material y esterilización del norte de Brasil}

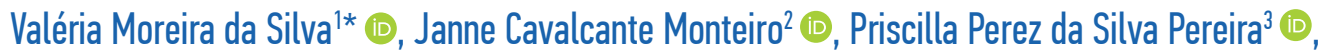 \\ Daniela Oliveira Pontes ${ }^{4}\left(\mathbb{C}\right.$, Ana Laura Salomão Pereira Fernandes ${ }^{5}$ (1)
}

RESUMO: Objetivo: Analisar o nível de riscos psicossociais dos trabalhadores do Centro de Material e Esterilização de um hospital de grande porte de Rondônia. Método: Estudo transversal, incluindo auxiliares, técnicos de enfermagem e enfermeiros, utilizando o questionário Copenhagen Psychosocial Questionnaire. As análises descritivas foram realizadas no pacote estatístico Stata ${ }^{\circledR}$ versão 11. Resultados: Participaram 35 trabalhadores, sendo a maioria mulheres, com idade acima de 40 anos e técnicas de enfermagem. Das seis dimensões avaliadas, quatro tiveram risco médio. A dimensão sobre justiça e respeito apresentou elevado risco psicossocial, e a dimensão organização do trabalho e conteúdo, baixo risco. Conclusão: Os trabalhadores consideravam seu trabalho importante e significante, porém vivenciavam alto risco psicossocial no que se refere à exigência de atenção e exigência emocional das atividades desenvolvidas na unidade. Poucos estavam satisfeitos com o trabalho, com o ambiente e com a utilização das habilidades individuais pelo serviço. As informações sobre as relações psicossociais constituem um importante indicador para análise das situações de trabalho, gerando informações que apoiam intervenções seguras sobre o processo de trabalho.

Palavras-chave: Impacto psicossocial. Equipe de enfermagem. Esterilização.

ABSTRACT: Objective: To analyze the level of psychosocial risks of workers at the Central Sterile Supply Department of a large hospital in Rondonia. Method: Cross-sectional study, including assistants, nursing technicians, and nurses, using the Copenhagen Psychosocial Questionnaire. Descriptive analyses were performed using the Stata ${ }^{\circledR}$ statistical package, version 11. Results: 35 workers took part in the study, most of them women, aged over 40 years old, and nursing technicians. Of the six dimensions assessed, four had a medium risk. The dimension of justice and respect presented a high psychosocial risk, and the dimension of work organization and content, low risk. Conclusion: The workers considered their work important and significant, but they experienced a high psychosocial risk with regard to the demand for attention and emotional demand of the activities carried out in the unit. Few were satisfied with the work, the environment, and the use of individual skills by the service. Information about psychosocial relationships is an important indicator for analyzing work situations, generating information that supports safe interventions in the work process.

Keywords: Psychosocial impact. Nursing, team. Sterilization.

RESUMEN: Objetivo: Analizar el nivel de riesgo psicosocial de los trabajadores del Centro de Material y Esterilización de un gran hospital de Rondônia. Método: Estudio transversal, incluyendo auxiliares, técnicos de enfermería y enfermeras, utilizando el Cuestionario Psicosocial de Copenhague. Los análisis descriptivos se realizaron con el paquete estadístico Stata ${ }^{\circledR}$ versión 11. Resultados: participaron 35 trabajadores, en su mayoría mujeres, mayores de 40 años y técnicos de enfermería. De las seis dimensiones evaluadas, cuatro tenían un riesgo medio. La dimensión de justicia y respeto presentó un alto riesgo psicosocial y la dimensión de organización y contenido del trabajo fue de bajo riesgo. Conclusión: Los trabajadores consideraron su trabajo importante y significativo, pero experimentaron un alto riesgo psicosocial en cuanto a la demanda de atención y demanda emocional de las actividades desarrolladas en la unidad. Pocos estaban satisfechos con el trabajo, el entorno y el uso de habilidades individuales por parte del servicio. La información sobre las relaciones psicosociales es un indicador importante para analizar situaciones laborales, generando información que sustente intervenciones seguras en el proceso laboral.

Palabras clave: Impacto psicosocial. Grupo de enfermería. Esterilización.

'Mestre em Ensino em Ciências da Saúde pela Universidade Federal de Rondônia (UNIR). Docente do Departamento de Enfermagem da UNIR - Porto Velho (RO), Brasil.

2Doutora em Engenharia de Produção pela Universidade Federal de Santa Catarina. Docente do Departamento de Medicina da UNIR - Porto Velho (RO), Brasil.

${ }^{3}$ Doutora em Ciência da Saúde pela Universidade de Brasília. Docente do Departamento de Enfermagem da UNIR - Porto Velho (RO), Brasil.

"Mestre em Biologia Experimental pela UNIR. Docente do Departamento de Enfermagem da UNIR - Porto Velho (RO), Brasil.

${ }^{5}$ Acadêmica do curso de Psicologia da UNIR - Porto Velho (RO), Brasil.

*Autora correspondente: valeria.moreira@unir.br

Recebido: 21/06/2020 - Aprovado: 08/01/2021

https://doi.org/10.5327/Z1414-4425202100010002 


\section{INTRODUÇÃO}

O Centro de Material e Esterilização (CME) é o setor que tem por atividade-fim fornecer produtos para a saúde (PPS) seguros para suprir a assistência direta ao usuário no Centro Cirúrgico, em unidades de internação, ambulatórios, setores de diagnóstico, além de unidades de urgências e emergências ${ }^{1-3}$.

É considerado uma área crítica, pois recepciona PPS contaminados com secreções orgânicas provenientes dos procedimentos realizados pela equipe de saúde. $\mathrm{O}$ ambiente e a organização do trabalho nesse setor expõem os trabalhadores a diversos riscos ocupacionais: biológicos, químicos (vapores e líquidos), físicos (ruídos, temperatura elevada e iluminação inadequada), ergonômicos (esforços físicos e posições incômodas) e psicossociais (estresse, insatisfação e sobrecarga mental $)^{4}$.

O processo de trabalho no CME exige dos trabalhadores habilidades e competências específicas em um ritmo de produção muito acelerado e com exigências físicas e mentais. Além do ambiente e do processo de trabalho, outros fatores podem potencializar problemas psicossociais entre os trabalhadores, como o suporte da gestão e os relacionamentos entre colegas e com o responsável técnico pelo setor ${ }^{5}$.

Uma revisão sistemática sobre a exposição a fatores de risco psicossocial em contextos de trabalho analisou 22 artigos que utilizaram o instrumento Copenhagen Psychosocial Questionnaire (COPSOQ) para mensuração dos fatores psicossociais. Os achados evidenciaram influência entre fatores individuais, fatores relacionados com o trabalho e fatores de risco psicossocial e que independem do risco exposto. Todos os trabalhadores incluídos nos estudos manifestavam prejuízos em relação à sua saúde mental e ao bem-estar físico ${ }^{6}$.

Um estudo conduzido no CME de um hospital universitário do Rio de Janeiro, com 34 profissionais, apontou que o ambiente físico, o processo e a organização do trabalho no setor estavam diretamente ligados ao desgaste físico e emocional dos profissionais? ${ }^{7}$. Os entrevistados relataram ser submetidos a condições laborais que ocasionavam estresse, diminuição da concentração e atenção, tendo como consequência o aparecimento de enxaqueca, hipertensão arterial ou úlcera gástrica ${ }^{7}$. Outro estudo realizado em um hospital público do estado de São Paulo, com 63 profissionais, mostrou que os principais fatores psicossociais que desencadeiam estresse e adoecimento no CME são a elevada demanda de trabalho, o relacionamento entre os membros da equipe, o suporte da gestão, o baixo reconhecimento e uma visão estigmatizada do setor por não trabalhar diretamente na assistência ao paciente 5 .

Na revisão de literatura prévia não se encontraram estudos sobre a temática na região amazônica. A Amazônia brasileira é constituída de nove estados, que ocupam $61 \%$ do território do país, abrigando $12 \%$ da população, que enfrenta diversos problemas de saúde pública, sociais e econômicos ${ }^{8}$. A instituição de saúde onde o estudo foi conduzido tem o desafio de processar os PPS com o mesmo padrão de qualidade de qualquer outra instituição, porém com problemas estruturais e de processo de trabalho peculiares a uma região distante dos grandes centros tecnológicos e de formação profissional.

\section{OBJETIVO}

Analisar o nível de riscos psicossociais dos trabalhadores de saúde que atuam no CME de um hospital geral público de Rondônia.

\section{MÉTODOS}

Trata-se de estudo transversal, realizado no CME de um hospital público de nível terciário, grande porte e referência estadual no provimento de assistência à saúde pública em Rondônia - contudo, recebe usuários do Amazonas, do Acre e da Bolívia ${ }^{8}$. Atende as especialidades de neurocirurgia, oncologia, cirurgia ortopédica, transplante de córneas e rins, cirurgia geral e cirurgia bariátrica. É o único serviço público de hemodinâmica do estado e também o único estabelecimento de assistência à saúde público que presta atendimento em Unidade de Terapia Intensiva Neonatal ${ }^{9}$.

O CME estudado é classificado como Classe II, de acordo com a Resolução da Diretoria Colegiada da Agência Nacional de Vigilância Sanitária $n^{\circ} 15$ de $2012^{3}$. Os PPS processados são utilizados nas diversas unidades do hospital. Esse CME é referência na etapa de esterilização dos PPS de outras instituições locais quando estas apresentam dificuldades operacionais na etapa de esterilização. 
O dimensionamento de pessoal foi observado pelas escalas de serviços mensais, sendo a média de oito profissionais de enfermagem de nível médio e um profissional de enfermagem de nível superior, exclusivo para o setor, numa jornada de 12 horas. Foram identificados 41 trabalhadores que atuavam diretamente no processamento de PPS, entre enfermeiros, técnicos e auxiliares de enfermagem, e todos foram convidados a participar do estudo. Foram incluídos os que estavam presentes no setor no período da coleta e excluídos aqueles em licença médica, férias ou licença-prêmio.

Do total de trabalhadores do CME, 35 participaram, sendo 29 auxiliares e técnicos de enfermagem e 6 enfermeiros. Seis foram excluídos por estarem de licença médica ou férias no período da coleta de dados, realizada entre março de 2016 e abril de 2017.

Foram utilizados dois questionários: o primeiro abordando aspectos sociodemográficos, ocupacionais e de hábitos de vida dos participantes, com 19 questões abertas e fechadas, baseadas no instrumento proposto por Santos ${ }^{10}$; o segundo foi o questionário COPSOQ, traduzido e adaptado por Silva et al. ${ }^{11}$, contendo 26 itens, com seis dimensões:

- exigências laborais;

- relações sociais e liderança;

- organização do trabalho e conteúdo;

- interface trabalho-indivíduo;

- justiça e respeito;

- saúde física geral.

Cada item continha cinco alternativas de resposta, com pontuação máxima de cinco e mínima de um ponto, sendo o risco final obtido por meio da média das respostas do item (Quadro 1).

A análise quantitativa foi realizada no programa Stata ${ }^{\circledR}$ versão 11. Os dados foram digitados e armazenados no Microsoft
Excel $^{\circledR}$, e foram calculadas as frequências absolutas e relativas e medidas de tendência central (média e desvio padrão [DP]).

Este estudo foi aprovado pelo Comitê de Ética em Pesquisa da Universidade Federal de Rondônia, sob parecer de aprovação 1.849 .750 .

\section{RESULTADOS}

Dentre os 35 participantes do estudo, obteve-se média de idade de 48 anos $(\mathrm{DP}=9,43)$. A maioria dos participantes apresentou as seguintes características: sexo feminino $(77,1 \%)$; pertencimento às categorias auxiliar e técnico de enfermagem (82,9\%); renda familiar de até quatro salários mínimos (60,0\%); tempo de formação superior a dez anos (74,3\%); tempo de serviço no setor maior que cinco anos (40,0\%); regime de plantão somando até 40 horas semanais $(94,9 \%)$; apenas um vínculo empregatício (60,0\%); execução apenas da função à qual foi designado no $\operatorname{CME}(85,7 \%)$. Aproximadamente metade dos profissionais atua nas áreas de preparo e esterilização de PPS (48,6\%), e $20,0 \%$ deles fazem horas extras com frequência (Tabela 1).

Para a maioria dos trabalhadores, o tempo de pausa na jornada de trabalho é menor do que 30 minutos $(62,9 \%)$, e a distribuição do período foi variável de acordo com o período do plantão. Pouco mais da metade dos participantes não realiza exercícios físicos (54,3\%), 22,9\% são ex-fumantes, e 5,7\% ainda fumam. Quanto ao Índice de Massa Corporal, a maioria apresentou as categorias sobrepeso ou obesidade (Tabela 2).

Em relação aos riscos psicossociais, de modo geral, a análise feita por meio do COPSOQ mostrou que a maioria das dimensões foi classificada como de médio risco. A dimensão justiça e respeito foi considerada de alto risco, e a organização do trabalho e conteúdo, de baixo risco psicossocial para os participantes (Tabela 3).

Quadro 1. Alternativas de resposta, pontuação e categorias do nível de risco psicossocial.

\begin{tabular}{|c|c|c|c|}
\hline Alternativas de resposta & $\begin{array}{c}\text { Pontuação } \\
\text { Questões } 1 \text { a } 8 \text { e } 22\end{array}$ & $\begin{array}{c}\text { Pontuação } \\
\text { Questões } 9 \text { a 25, exceto } 22\end{array}$ & Categorias/média \\
\hline Sempre & 5 & 1 & \multirow{5}{*}{$\begin{array}{l}\text { Alto risco: maior que } 3,67 \\
\text { Médio risco: } 2,35 \text { a } 3,66 \\
\text { Baixo risco: menor que } 2,34\end{array}$} \\
\hline Com frequência & 4 & 2 & \\
\hline Algumas vezes & 3 & 3 & \\
\hline Raramente & 2 & 4 & \\
\hline Nunca & 1 & 5 & \\
\hline
\end{tabular}


Tabela 1. Perfil sociodemográfico e ocupacional dos profissionais participantes da pesquisa, que atuam no Centro de Material e Esterilização ( $n=35)$.

\begin{tabular}{|c|c|c|c|c|c|}
\hline Variável & $\mathbf{N}$ & $\%$ & Variável & $\mathbf{N}$ & $\%$ \\
\hline Sexo & & & Área em que atua & & \\
\hline Feminino & 27 & 77,1 & Área de recepção e limpeza & 10 & 28,6 \\
\hline Masculino & 08 & 22,9 & Área de preparo e esterilização & 17 & 48,6 \\
\hline Categoria profissional & & & Área de armazenamento & 08 & 22,8 \\
\hline Auxiliar/técnico de enfermagem & 29 & 82,9 & Outra função no Centro de Material e Esterilização & & \\
\hline Enfermeiro & 06 & 17,1 & Não & 30 & 85,7 \\
\hline Faixa etária (anos) & & & Sim & 05 & 14,3 \\
\hline 26 a 30 & 02 & 5,7 & Jornada de trabalho & & \\
\hline 31 a 35 & 02 & 5,7 & Plantonista & 29 & 82,9 \\
\hline 36 a 40 anos & 05 & 14,3 & Diarista & 06 & 17,1 \\
\hline 41 a 45 & 06 & 17,2 & Carga horária no setor (horas) & & \\
\hline 46 a 50 & 02 & 5,7 & $\leq 40$ & 33 & 94,9 \\
\hline Acima de 50 & 18 & 51,4 & $>40$ & 02 & 5,1 \\
\hline Renda familiar* (salário mínimo) & & & Mais de um vínculo empregatício & & \\
\hline $0-4$ & 21 & 60,0 & Não & 21 & 60,0 \\
\hline $5-10$ & 09 & 25,7 & Sim & 14 & 40,0 \\
\hline$>10$ & 05 & 14,3 & Com frequência faz horas extras & & \\
\hline Tempo de profissão (anos) & & & Não & 28 & 80,0 \\
\hline $0-4$ & 03 & 8,6 & Sim & 07 & 20,0 \\
\hline $5-9$ & 06 & 17,1 & Período de pausa & & \\
\hline$\geq 10$ & 26 & 74,3 & Manhã & 12 & 34,3 \\
\hline Tempo no Centro de Material e Esterilização & & & Tarde & 09 & 25,7 \\
\hline (anos) & & & Noite & 14 & 40,0 \\
\hline $0-4$ & 10 & 28,6 & Duração da pausa (minutos) & & \\
\hline $5-9$ & 14 & 40,0 & $\leq 30$ & 22 & 62,9 \\
\hline$\geq 10$ & 11 & 31,4 & $>30$ & 13 & 37,1 \\
\hline
\end{tabular}

*Valor do salário mínimo no Brasil no ano de 2017=R\$ 937,00, segundo o Departamento Intersindical de Estatística e Estudos Socioeconômicos ${ }^{2}$.

Tabela 2. Estilo de vida dos profissionais participantes da pesquisa, que atuam no Centro de Material e Esterilização ( $n=35)$.

\begin{tabular}{|l|c|c|}
\hline Variável & N & $\%$ \\
\hline Exercícios físicos & 19 & 54,3 \\
\hline Não & 16 & 45,7 \\
\hline Sim & & \\
\hline Fumo & 25 & 71,4 \\
\hline Não, nunca fumei & 08 & 22,9 \\
\hline Não, ex-fumante & 02 & 5,7 \\
\hline Sim & & \\
\hline Índice de Massa Corporal & - & - \\
\hline Baixo peso & 10 & 28,6 \\
\hline Eutrófico & 15 & 42,9 \\
\hline Sobrepeso & 08 & 22,9 \\
\hline Obesidade I & - & - \\
\hline Obesidade II & 02 & 5,6 \\
\hline Obesidade III & & \\
\hline
\end{tabular}

Nessa análise, foram encontrados altos riscos nas seguintes categorias:

- Exigências laborais, exigência emocional de si (média $=3,94 ; \mathrm{DP}=1,21$ ) e cognitiva, no que se refere à exigência de atenção constante na execução do trabalho (média $=4,82 ; \mathrm{DP}=0,71$ );

- Relações sociais e liderança, no que se refere ao fato de os colegas não estarem abertos a ouvir sobre os problemas de trabalho (média $=3,71 ; \mathrm{DP}=0,99$ );

- Justiça e respeito, quanto ao sentimento de pertencimento de uma comunidade (média $=4,00 ; \mathrm{DP}=1,37$ ).

Os itens considerados com baixo risco foram:

- Relações sociais e liderança, no quesito ajuda e apoio dos colegas de trabalho (média $=2,25 ; \mathrm{DP}=0,95$ );

- Organização do trabalho e conteúdo, no que se refere ao significado do trabalho para si (média $=1,86$; $\mathrm{DP}=0,49)$ e o sentimento de que o trabalho é importante (média $=1,82 ; \mathrm{DP}=0,45$ ).

A percepção dos participantes quanto à sua saúde geral foi classificada como boa (média $=3,00 ; \mathrm{DP}=1,03$ ), porém um participante considerou sua saúde excelente, e três como deficiente. 
Tabela 3. Nível de riscos psicossociais dos profissionais participantes da pesquisa, que atuam no Centro de Material e Esterilização $(n=35)$.

\begin{tabular}{|c|c|c|c|c|c|c|c|c|c|c|c|}
\hline \multirow[t]{2}{*}{ Variável } & \multicolumn{2}{|c|}{ Sempre } & \multicolumn{2}{|c|}{$\begin{array}{c}\text { Com } \\
\text { frequência }\end{array}$} & \multicolumn{2}{|c|}{$\begin{array}{l}\text { Algumas } \\
\text { vezes }\end{array}$} & \multicolumn{2}{|c|}{ Raramente } & \multicolumn{2}{|c|}{ Nunca } & \multirow[t]{2}{*}{$\begin{array}{c}\text { Média (DP) / Nível } \\
\text { de risco }\end{array}$} \\
\hline & $\mathbf{N}$ & $\%$ & $\mathbf{N}$ & $\%$ & $\mathbf{N}$ & $\%$ & $\mathbf{N}$ & $\%$ & $\mathbf{N}$ & $\%$ & \\
\hline \multicolumn{11}{|c|}{ Exigências laborais } & $3,23(0,83)$ médio \\
\hline \multicolumn{12}{|l|}{ Quantitativa } \\
\hline 1. Carga horária mal distribuída & 02 & 5,71 & 07 & 20,00 & 10 & 28,57 & 07 & 20,00 & 09 & 25,71 & $2,60(1,24)$ médio \\
\hline $\begin{array}{l}\text { 2. Não tem tempo para completar } \\
\text { todas as tarefas }\end{array}$ & 08 & 14,29 & 01 & 2,86 & 07 & 20,00 & 14 & 40,00 & 08 & 22,86 & $2,46(1,29)$ médio \\
\hline $\begin{array}{l}\text { 3. Precisa fazer horas extras } \\
\text { Ritmo de trabalho }\end{array}$ & 07 & 20,00 & 08 & 22,86 & 11 & 31,43 & 02 & 5,71 & 07 & 20,00 & $3,17(1,38)$ médio \\
\hline $\begin{array}{l}\text { 4. Precisa trabalhar muito } \\
\text { rapidamente }\end{array}$ & 04 & 11,43 & 05 & 14,29 & 09 & 25,71 & 07 & 20,00 & 10 & 28,57 & 2,60 $(1,35)$ médio \\
\hline \multicolumn{12}{|l|}{ Emocionais } \\
\hline $\begin{array}{l}\text { 5. Exige emocionalmente de si } \\
\text { Cognitivas }\end{array}$ & 16 & 45,71 & 08 & 22,86 & 05 & 14,29 & 05 & 14,29 & 01 & 2,86 & $3,94(1,21)$ alto \\
\hline 6. Exige atenção constante & 32 & 91,43 & 02 & 5,71 & - & - & - & - & 01 & 2,86 & $4,82(0,71)$ alto \\
\hline $\begin{array}{l}\text { 7. Trabalho requer que seja bom } \\
\text { para propor novas ideias }\end{array}$ & 10 & 28,57 & 12 & 34,29 & 05 & 14,29 & 06 & 17,14 & 02 & 5,71 & 3,63 $(1,24)$ médio \\
\hline $\begin{array}{l}\text { 8. Trabalho exige que tome } \\
\text { decisões difíceis }\end{array}$ & 03 & 8,57 & 05 & 14,29 & 11 & 31,43 & 10 & 28,57 & 06 & 17,14 & 2,68 $(1,18)$ médio \\
\hline \multicolumn{11}{|c|}{ Relações sociais e liderança } & $3,08(0,58)$ médio \\
\hline \multicolumn{12}{|l|}{ Apoio social de colegas } \\
\hline $\begin{array}{l}\text { 9. Tem ajuda e apoio dos seus } \\
\text { colegas de trabalho }\end{array}$ & 09 & 25,71 & 11 & 31,43 & 12 & 34,29 & 03 & 22,86 & 09 & 25,71 & 2,25 (0,95) baixo \\
\hline $\begin{array}{l}\text { 10. Colegas ouvem sobre os seus } \\
\text { problemas de trabalho }\end{array}$ & 05 & 14,29 & 05 & 14,29 & 11 & 31,43 & 09 & 25,71 & 05 & 14,29 & $3,71(0,99)$ alto \\
\hline $\begin{array}{l}\text { 11. Colegas falam a respeito do seu } \\
\text { desempenho laboral }\end{array}$ & & - & 04 & 11,43 & 11 & 31,43 & 11 & 31,43 & 09 & 25,71 & \\
\hline \multicolumn{12}{|l|}{ Apoio social de superiores } \\
\hline $\begin{array}{l}\text { 12. Superior imediato fala sobre } \\
\text { como está o seu trabalho }\end{array}$ & 05 & 14,29 & 03 & 8,57 & 8 & 22,86 & 12 & 34,29 & 07 & 20,00 & 3,37 $(1,31)$ médio \\
\hline $\begin{array}{l}\text { 13. Tem ajuda e apoio do seu } \\
\text { superior imediato }\end{array}$ & 10 & 28,57 & 07 & 20,00 & 9 & 25,71 & 08 & 22,86 & 01 & 2,86 & $2,51(1,22)$ médio \\
\hline $\begin{array}{l}\text { 14. Superior imediato fala sobre } \\
\text { seu desempenho laboral }\end{array}$ & 04 & 11,43 & 03 & 8,57 & 5 & 14,29 & 17 & 48,57 & 06 & 17,14 & 3,51 $(1,22)$ médio \\
\hline
\end{tabular}

Organização do trabalho e conteúdo

$2,17(0,58)$ baixo

Significado do trabalho

15. 0 seu trabalho tem algum significado para si

\begin{tabular}{|l|l|l|l|l|l|l|l|l|l|l|}
\hline 07 & 20,00 & 26 & 74,29 & 02 & 5,71 & - & - & - & - & $1,86(0,49)$ baixo \\
\hline 07 & 20,00 & 27 & 77,14 & 01 & 2,86 & - & - & - & - & $1,82(0,45)$ baixo \\
\hline 01 & 2,86 & 12 & 34,29 & 13 & 37,14 & 09 & 25,71 & - & - & $2,85(0,84)$ médio \\
\hline
\end{tabular}
importante

17. Sente-se motivado e envolvido com o seu trabalho

Satisfação do trabalho

18. Satisfeito com as perspectivas de trabalho

19. Satisfeito com o seu trabalho de uma forma global 20. Satisfeito com as condições físicas do local 21. Satisfeito com a forma como as suas capacidades são utilizadas Insegurança laboral 22. Sente-se preocupado em ficar desempregado

\begin{tabular}{|c|c|c|c|c|c|c|c|c|c|c|}
\hline 01 & 2,86 & 18 & 51,43 & 13 & 37,14 & 03 & 51,43 & - & - & $2,51(0,70)$ médio \\
\hline 02 & 5,71 & 07 & 20,00 & 15 & 42,86 & 11 & 31,43 & - & - & $3,00(0,87)$ médio \\
\hline 02 & 5,71 & 15 & 42,86 & 17 & 48,57 & 01 & 2,86 & - & - & $2,48(0,66)$ médio \\
\hline- & - & 06 & 17,14 & 17 & 48,57 & 12 & 34,29 & - & - & $3,17(0,71)$ médio \\
\hline 13 & 37,14 & 07 & 20,00 & 02 & 5,71 & 10 & 28,57 & 03 & 8,57 & $2,51(1,46)$ médio \\
\hline
\end{tabular}


Tabela 3. Continuação

\begin{tabular}{|c|c|c|c|c|c|c|c|c|c|c|c|c|c|}
\hline \multirow{2}{*}{\multicolumn{3}{|c|}{ Variável }} & \multicolumn{2}{|c|}{ Sempre } & \multicolumn{2}{|c|}{$\begin{array}{c}\text { Com } \\
\text { frequência } \\
\end{array}$} & \multicolumn{2}{|c|}{$\begin{array}{c}\text { Algumas } \\
\text { vezes }\end{array}$} & \multicolumn{2}{|c|}{ Raramente } & \multicolumn{2}{|c|}{ Nunca } & \multirow[t]{2}{*}{$\begin{array}{c}\text { Média (DP) / Nível } \\
\text { de risco }\end{array}$} \\
\hline & & & $\mathbf{N}$ & $\%$ & $\mathbf{N}$ & $\%$ & $\mathbf{N}$ & $\%$ & $\mathbf{N}$ & $\%$ & $\mathbf{N}$ & $\%$ & \\
\hline \multicolumn{13}{|c|}{ Justiça e respeito } & $3,69(0,27)$ alto \\
\hline \multicolumn{14}{|c|}{ Comunidade social no trabalho } \\
\hline \multicolumn{3}{|c|}{$\begin{array}{l}\text { 23. Existe um bom ambiente de } \\
\text { trabalho entre si e os seus colegas }\end{array}$} & - & - & 05 & 14,29 & 13 & 37,14 & 09 & 25,71 & 08 & 22,86 & $3,57(1,00)$ médio \\
\hline \multicolumn{3}{|c|}{$\begin{array}{l}\text { 24. Existe uma boa cooperação } \\
\text { entre os colegas de trabalho }\end{array}$} & - & - & 04 & 11,43 & 15 & 42,86 & 10 & 28,57 & 06 & 17,14 & $3,51(0,92)$ médio \\
\hline \multicolumn{3}{|c|}{$\begin{array}{l}\text { 25. No seu local de trabalho, } \\
\text { sente-se parte de uma comunidade }\end{array}$} & 02 & 5,71 & 06 & 17,14 & 2 & 5,71 & 05 & 14,29 & 20 & 57,14 & $4,00(1,37)$ alto \\
\hline \multicolumn{13}{|c|}{ Saúde física geral } & $3,00(1,03)$ médio \\
\hline & Excelente & $\%$ & $\begin{array}{c}\text { Muito } \\
\text { boa }\end{array}$ & $\%$ & Boa & $\%$ & \multicolumn{2}{|c|}{ Razoável } & $\%$ & \multicolumn{2}{|c|}{ Deficiente } & $\%$ & \\
\hline 26. Saúde geral & 01 & 2,86 & 12 & 34,29 & 11 & 31,43 & \multicolumn{2}{|c|}{08} & 22,86 & \multicolumn{2}{|l|}{03} & 8,57 & $3,00(1,03)$ médio \\
\hline
\end{tabular}

\section{DISCUSSÃO}

Os resultados do estudo evidenciaram que os riscos psicossociais influenciam no processo de trabalho no CME e, consequentemente, predispõem os trabalhadores a condições de adoecimento. Observa-se, na revisão de literatura da temática, déficit de estudos realizados na Amazônia sobre o fator psicossocial, dada a peculiaridade do processo de trabalho no CME, focado no sistema de produção de PPS com uma organização de trabalho baseada numa divisão sociotécnica e fragmentada.

O perfil sociodemográfico e profissional dos participantes foi de sexo feminino, com idade acima de 50 anos e cargos de nível médio (Tabela 1). Esses dados corroboram o perfil de trabalhador comumente lotado no CME, ou seja, profissionais prestes a aposentarem-se, que estão mais propensos a sentirem-se desmotivados, apresentarem desgastes físicos e emocionais ${ }^{4,5,13}$. Além disso, o gênero feminino parece ser um precursor para maiores níveis de estresse e a idade avançada é fator de risco para tarefas mais pesadas, no que se refere à resistência e à resiliência 6 .

A maioria tinha renda familiar de até quatro salários mínimos, trabalhava em regime de plantão, 40,0\% tinham mais de um vínculo empregatício, e 20,0\% faziam horas extras com frequência (Tabela 1). Mais de um emprego ou trabalhos extras podem trazer compensação financeira, porém muitas horas de trabalho podem levar a sobrecarga e jornadas extenuantes, interferindo nos processos de trabalho e contribuindo para o adoecimento dos trabalhadore ${ }^{13,14}$. O período de pausa durante as atividades estava condicionado ao quantitativo de trabalhadores escalados para a jornada e à demanda de recebimento de PPS para processamento. Um estudo sobre os riscos ergonômicos no CME, realizado em um hospital público do Piauí, demonstrou que a permanência em uma mesma postura corporal sem pausas por longo tempo acarreta, além dos distúrbios osteomusculares, distúrbios psicológicos ${ }^{15}$.

Outros riscos psicossociais relacionados às longas jornadas de trabalho são: diminuição da capacidade de atenção, concentração, percepção e tomada de decisão; desenvolvimento de estresse; ansiedade, depressão e apatia; abuso de substâncias, como álcool, tabaco e outras drogas ${ }^{6,7}$. A exposição a esses riscos eleva o nível de estresse dos trabalhadores e, por consequência, pode levar ao uso de substâncias como o álcool e o tabaco. Também desencadeia aspectos fisiológicos, como reações neuroendócrinas e imunológicas, que podem estar associadas ao ganho de peso e à menor disposição para a prática de exercícios físicos ${ }^{6}$. Esses três fatores são riscos para doenças cardiovasculares e interferem no estado geral de saúde.

Além dos hábitos de vida, o cotidiano estressante no trabalho colabora para que os trabalhadores consumam uma alimentação mais calórica do que o organismo necessita, geralmente pobre em fibras e nutrientes, o que pode relacionar-se com o sobrepeso ou a obesidade. Em Rondônia, a maioria dos trabalhadores encontrava-se com peso acima de eutrófico (Tabela 2); menos da metade praticava exercícios físicos; $22,9 \%$ haviam sido fumantes; 5,7\% relataram fumar.

Na dimensão exigências laborais, encontraram-se a maior média para risco psicossocial no item exigência de atenção constante durante o trabalho e, nessa mesma dimensão, alto risco no item exigência emocional de si mesmo (Tabela 3). Essa elevada exigência de atenção deve-se, principalmente, à sofisticação, às novas conformações dos PPS e aos avanços tecnológicos dos métodos de esterilização e abordagens cirúrgicas ${ }^{16}$. 
Relações sociais e liderança teve risco médio para problemas psicossociais (Tabela 3). Foram encontrados baixo risco para ajuda dos colegas e alto risco para o item colegas ouve sobre seus problemas de trabalho, e $57,14 \%$ dos participantes assinalaram que raramente ou nunca tiveram diálogo a respeito do desempenho laboral. Essa ausência de diálogo sobre o desempenho laboral por parte da gestão pode estar relacionada à desvalorização do trabalho desempenhado no setor em comparação àqueles que prestam assistência direta ao paciente. Entretanto, o produto final das atividades realizadas no CME contribui para a continuidade do cuidado de saúde em toda a cadeia de assistência ${ }^{4}$.

Em Rondônia, encontrou-se baixo risco psicossocial para a dimensão organização do trabalho e conteúdo. O trabalho foi avaliado como significante e importante para a maioria dos trabalhadores, e a maior parte dos participantes indicou ter ajuda dos seus colegas (Tabela 3).

A dimensão interface trabalho-indivíduo apresentou médio risco psicossocial. Mais da metade dos profissionais demonstrou preocupação com sua permanência no emprego, o que pode ser explicado pelo contrato de trabalho temporário de alguns. Menos da metade dos trabalhadores estavam satisfeitos, de forma geral, com seu trabalho e com as condições do ambiente, e apenas $17,1 \%$ sentiam-se satisfeitos com a forma com que suas capacidades eram utilizadas no setor. Um estudo conduzido em dez hospitais da rede pública em São Paulo e em um hospital da rede privada de Belo Horizonte (MG), com 463 participantes, encontrou que o sentido do trabalho para aqueles profissionais era a possibilidade de valorização profissional e a melhoria socioeconômica, e os profissionais do hospital privado apresentaram maior comprometimento afetivo com o seu trabalho, em razão da instabilidade do vínculo empregatício ${ }^{17}$.

Na dimensão comunidade social do trabalho, quase metade dos trabalhadores indicaram que o ambiente de trabalho raramente ou nunca é bom, e, no quesito cooperação entre os colegas, pouco menos da metade indicou que raramente ou nunca existe boa cooperação (Tabela 3). Isso demonstra uma probabilidade de conflitos relacionais no ambiente laboral, podendo acarretar estresse e desgastes psicológicos nos trabalhadores. Corroborando esse achado, um estudo realizado em hospital universitário do estado de São Paulo, com 63 participantes, concluiu que os principais fatores psicossociais no CME estudado são a elevada demanda de trabalho, agravada pelo absenteísmo de colegas, pelo trabalho em ambiente fechado e sem comunicação com os demais setores, e a desvalorização do trabalho por parte dos demais profissionais do hospital ${ }^{5}$.
Encontrou-se, em geral, médio risco psicossocial (Tabela 3), porém alto risco na dimensão justiça e respeito, no item sentir-se parte da comunidade. Ou seja, os trabalhadores do estudo não se sentiam parte de grupo, o que é essencial para o trabalho colaborativo e contrapõe-se às atividades propostas no CME.

Além disso, no domínio relações sociais e liderança, observou-se alto risco no item que avaliou o quanto os colegas são receptivos a ouvir os problemas do indivíduo no trabalho. Trabalho em equipe é vital nessa atividade, uma vez que os processos são sequenciais e somativos, e a repercussão no caso de falhas poderá gerar danos graves.

Como limitação do estudo, considera-se a não avaliação das implicações das relações psicossociais e de possíveis desfechos no processo saúde-doença dos trabalhadores do CME.

\section{CONSIDERAÇÕES FINAIS}

Neste estudo, os trabalhadores consideravam seu trabalho importante e significante, porém vivenciavam alto risco psicossocial no que se refere à exigência de atenção e à exigência emocional das atividades desenvolvidas no CME. Poucos estavam satisfeitos com o trabalho, com o ambiente e com a utilização das habilidades individuais pelo serviço.

As informações sobre as relações psicossociais constituem importante indicador para a compreensão sobre o processo de trabalho no CME. O olhar para esse grupo precisa ser repensado pelos gestores e pelas organizações de cuidado à saúde, pois compreender os processos de trabalho na dinâmica do CME contribui para uma organização não apenas centrada no produto, mas que considere o trabalhador como sujeito determinante para a melhor qualidade do processamento de PPS.

Os riscos psicossociais apresentaram e refletiram condições indesejadas para a atualidade e para o futuro de uma prática centrada na segurança do paciente e na prevenção de infecções relacionadas à assistência à saúde e livre de eventos adversos.

Consideram-se relevante realizar ações de sensibilização para o cuidado à saúde do trabalhador e desenvolver estratégias de Educação Permanente em Saúde, com ações intersetoriais com o Núcleo de Educação Permanente e o Núcleo de Vigilância dos Agravos à Saúde do Trabalhador.

Destaca-se, ainda, que o CME do estudo é cenário de referência para as práticas de ensino dos estudantes de enfermagem em nível superior e técnico, contribuindo para o ensino e para pesquisas relacionadas à saúde do trabalhador nas instituições de saúde. 


\section{REFERÊNCIAS}

1. Costa JA, Fugulin FMT. Atividades de enfermagem em centro de material e esterilização: contribuição para o dimensionamento de pessoal. Acta Paul Enferm. 2011;24(2):249-56. https://doi.org/10.1590/ S0103-21002011000200015

2. Gil RF, Camelo SH, Laus AN. Atividades do enfermeiro de centro de material e esterilização em instituições hospitalares. Revista Texto Contexto Enferm. 2013;22(4):927-34. https://doi.org/10.1590/ S0104-07072013000400008

3. Associação Brasileira de Enfermeiros de Centro Cirúrgico, Recuperação Anestésica e Central de Material e Esterilização (SOBECC). Diretrizes de práticas em enfermagem cirúrgica e processamento de produtos para a saúde. 7a ed. Barueri: Manole; São Paulo: SOBECC; 2017.

4. Rego GMV, Rolim ILTP, D'Eça Jr. A, Sardinha AHL, Lopes GSG, Coutinho NPS. Qualidade de vida no trabalho numa central de materiais e esterilização. Rev Bras Enferm. 2020;73(2):1-7. https:// doi.org/10.1590/0034-7167-2018-0792

5. Guissi PC, Pinho MASZ, Vieira I, Ranali Neto F, Martins DA, Bandini $M C D$, et al. Os fatores psicossociais no trabalho e estresse entre os profissionais de enfermagem de uma central de materiais esterilizados. Rev Bras Med Trabalho. 2019;17(4):449-505. https://doi.org/10.5327/ Z1679443520190453

6. Fernandes C, Pereira A. Exposição a fatores de risco psicossocial em contexto de trabalho: revisão sistemática. Rev Saúde Pública. 2016;50:1-15. https://doi.org/10.1590/S1518-8787.2016050006129

7. Costa CCP, Souza NVDO, Silva PAS, Oliveira EB, Vieira MLC. 0 trabalho na central de material: repercussões para a saúde dos trabalhadores de enfermagem. Rev Enferm UERJ. 2015;23(4):533-9. https://doi. org/10.12957/reuerj.2015.15934

8. Brasil. Ministério da Saúde. Cadastro Nacional de Estabelecimentos de Saúde. DATASUS [acessado em 20 dez 2019]. Brasil: Ministério da Saúde. Disponível em: http://cnes2.datasus.gov.br/cabecalho_ reduzido.asp?VCoc_Unidade $=1100204001303$

9. Rondônia. Secretaria de Estado da Saúde. Relatório de Gestão 2016 : Resultados e Perspectivas. Porto Velho: Secretaria de Estado da Saúde; 2016.438 p.

10. Santos AIS. Usabilidade dos equipamentos de proteção individual radiológica: pesquisa com técnicos e enfermeiros do CHLC [dissertação online]. Lisboa: Universidade de Lisboa, 2014 [acessado em 5 abr. 2017]. Disponível em: https://www.repository.utl.pt/ bitstream/10400.5/6911/1/Universidade $\% 20$ de $\% 20$ Lisboa\%20 $21 \% 20$ Maio\%20final.pdf

11.Silva C, Amaral V, Pereira A, Bem-haja P, Pereira A, Rodrigues V, et al. Copenhagen Psychosocial Questionnaire [Internet]. Universidade de Aveiro: Análise Exacta; 2012 [acessado em 20 set. 2016]. Disponível em: http://aciff.pt/uploads/Copenhagen\%20 psychosocial\%20questionnarie_COPSOQ.pdf

12. Departamento Intersindical de Estatística e Estudos Socioeconômicos (DIESSE). Nota Técnica n 166. Política de Valorização do Salário Mínimo: depois de 20 anos, reajuste fica abaixo da inflação (INPC) [Internet]. Departamento Intersindical de Estatística e Estudos Socioeconômicos; 2017 [acessado em 10 dezembro de 2020] Disponível em: https://www.dieese.org. br/analisecestabasica/salarioMinimo.html\#2017

13. Costa CCP, Souza NVDO, Pires AS. Perfil dos trabalhadores de uma central de material e esterilização: uma análise das características sócio-profissionais. Rev Pesq Cuidado Fundamental Online 2016;8(1):3633-45. https://doi. org/10.9789/2175-5361.2016.v8i1.3633-3645

14.Spagnol CA, Oliveira BKS, Candian ES, Santos RO, Manoel VCF, Moreira AR. 0 jogo como estratégia de promoção de qualidade de vida no trabalho no centro de material e esterilização. Rev Enferm Cent O Min. 2015;5(2):1562-73. https://doi.org/10.19175/ recom.v0i0.1064

15.Gouveia MTO, Oliveira VC, Lira IMS. Riscos ergonômicos em um centro de material e esterilização. Rev Enferm UFPI [Internet]. 2016 [acessado em 24 de dezembro de 2019];5(3):42-7. Disponível em: https://pesquisa.bvsalud.org/portal/resource/ pt/bde-31842

16. Borgheti SP, Viegas K, Caregnato RCA. Biossegurança no centro de materiais e esterilização: dúvidas dos profissionais. Rev SOBECC. 2016;21(1):3-12. https://doi.org/10.5327/ Z1414-4425201600010002

17. Rodrigues AL, Barrichello A, Morin EM. Os sentidos do trabalho para profissionais de enfermagem: um estudo multimétodos. Rev Adm Empres. 2016;56(2):192-208. https://doi.org/10.1590/ S0034-759020160206 